\title{
36. THEORY OF STELLAR ATMOSPHERES (THÉORIE DES ATMOSPHĖRES STELLAIRES)
}

PRESIDENT: R. Cayrel

VICE-PRESIDENT: D. Mihalas

ORGANIZING COMMITTEE: E. H. Avrett, K. Hunger, J. Hutchings, V. L. Kholklova, B. E. J. Pagel, F. Praderie, R. N. Thomas, M. Vardya

\section{INTRODUCTION, COMMISSION ACTIVITY AND COLLOQUIA OR SYMPOSIA IN THE FIELD OF THE THEORY OF STELLAR ATMOSPHERES}

This report has been prepared with the active cooperation of all members of the Organizing Committee of the Commission. It is based on the analysis of over 600 references dealing with the subject of stellar atmospheres.

The commission has scientifically sponsored or cosponsored the following colloquia (1) IAU Symposium No. 73: 'Abundance Effects in Classification', Lausanne (July 8 to 12, 1975), (2) International Colloquium of the Centre National de la Recherche Scientifique, 'Physics of Motions in Stellar Atmospheres', Nice (September 1 to 5, 1975) and (3) IAU Symposium No. 70 on 'Be and Shell Stars' (September 15 to 19, Cape Cod, 1975). Not sponsored by Commission 36 but worth noting is the IAU Colloquium No. 32, on 'Physics of Ap Stars', Vienna (September 8 to 10,1975 ).

Commission 36 also proposed a joint discussion, for the 1976 General Assembly on the topic 'Stellar atmospheres as factor and indicator of stellar evolution' with the following subtopics in mind: mass loss, diffusion processes and mixing of interior with surface layers in advanced stages of evolution.

The report is organized in the following manner: first it gives (Section 2) a very brief outline of the main developments which have occurred during the years 1973-1975. This outline is written for astronomers who are not specialists in the field and may be of some use to the others as well.

The following sections deal with specific areas of the field, going from general methods to problems of decreasing generality. They are more technical and end with an account of works dealing with particular objects, most of them having for chief aim the determination of the elemental abundances using the technique of model stellar atmospheres.

The bibliography starts with general references and is then ordered by topics.

\section{BRIEF OUTLINE OF THE MAIN DEVELOPMENTS IN THE FIELD OF STELLAR ATMOSPHERES}

Phenomena currently described as 'diffusion processes' (but dealing with several kinds of selective forces on different atomic species) have been shown to play an important role in stellar atmospheres and to be the most plausible source of explanation for several abundance 'anomalies', for the Ap stars in particular and for other related peculiarities in earlior types. Interconnected with this subject is our understanding of the dynamical state of stellar atmospheres. The explanation, 15 years after their discovery, of the semi-periodic motions in the solar photosphere (the so-called five minute oscillations) as high horizontal modes of the first few radial modes of vibration of the sun, is a large step in this area.

Great advances have been realized also in a self-consistent treatment of the transfer of radiation in extended (and expanding) atmospheres including non-LTE effects, and the dynamic effects of radiation on the matter of hot stars. Quantitative models have been 
produced which give great hope of helping us to understand the generality of mass loss and the existence of chromospheres and coronae in hot stars, as evidenced by Copernicus observations in the 1100-1500 $\AA$ UV range. General solutions of the non-LTE transfer equations in the presence of a stochastic velocity field have now been obtained.

In cool stars great efforts have been made to produce models including the contribution of molecules to the opacity via 'opacity distribution functions.' Also the formation of dust grains (nucleation) and their subsequent effect on the opacity and mass loss rate in cool giants and supergiants have been the subject of several investigations. Theoretical works have suggested that coronae of giants and supergiants could make a significant contribution to the soft X-ray galactic emission, but the recent observations from space of nearby stars do not give much support to these models.

The existence of a large spread in the damping constants of lines in the solar curve of growth has been shown to have strong implications on the accepted value of the solar 'microturbulence', and to be the cause of spurious gravities in differential analyses with respect to the sun, when an average curve of growth is used. The number of papers on stellar atmospheres using a non-LTE approach now significantly exceeds the number of papers using the LTE approach.

It has recently been show $\mathrm{n}$ that in making an accurate comparison of theoretical profiles with observations one needs to take into account the partial redistribution produced by Doppler motions instead of the frequently-used complete redistribution assumption. This is of special importance far the $\mathrm{K}_{1}$ component of $\mathrm{Ca}$ II resonance line. Abundance analyses through stellar atmospheres techniques have been performed for several hundreds of stars; often these allow for departures from LTE. One conspicuous result is the removal of the long-standing discrepancy between the neon abundance in B stars and in the solar system. The larger abundance usually found in LTE analyses of B stars has been shown spurious, a non-LTE analysis brings the mean abundance down to its solar system value.

\section{RADIATIVE TRANSFER, THEORY OF SPECTR UM FORMATION IN A PLANE-PARALLEL ATMOSPHERE}

\section{A. The Non-LTE Transfer Equation}

An important new formulation of the so-called complete linearization method for solving the transfer equation coupled to the equations of statistical equilibrium (non-LTE), has been given by Auer and Heasley (1976). Their method has very favorable computer-time scaling properties for cases where large numbers of frequencies in the spectrum must be considered. Kalkofen (1974) has developed an integralequation version of the complete linearization method.

\section{B. Partial Redistribution Effect on Line Formation}

Practically all astrophysical analyses of stellar spectra in which departures from LTE are taken into account are based upon the simplifying assumption of complete frequency redistribution in the scattering process, despite the fact that it is known that this assumption is not correct in many regimes. Vernazza et al. (1973) showed that to fit the observed solar Lyman $\alpha$ profile it is necessary to suppose that the scattering in the line wing has a high degree of coherence. Milkey and Mihalas (1973a, 1973b) were able to fit the profile quantitatively by allowing for partial redistribution function. Further work has demonstrated important effects upon the computed profiles of the solar Mg II H and K lines (Milkey and Mihalas, 1974), stellar Mg II H and K lines (Milkey, Ayres and Shine, 1975) the solar Ca II H and K lines (Vardavas and Cram, 1974; Milkey, Shine and Mihalas, 1975a, and Shine, Milkey and Mihalas, 1975a), and on stellar Ca II H and $\mathrm{K}$ lines (Shine, Milkey and Mihalas, 1975b). The agreement of computed and observed limb-darkening of the Ca II lines is much improved when partial redistribution is allowed. A study of angular effects by Milkey, Shine and Mihalas (1975b) indicates that for homogeneous, static atmosphere it is sufficient to use angle-averaged redistribution functions for the Ca II 
lines. Heasley and Kneer (1975) have developed a simple and economical method to treat partial redistribution effects. Partial redistribution effects in the presence of velocity fields will be discussed in section 4 below. The work done thus far all indicates that, relative to complete redistribution, the effects of partial redistribution decrease the intensity in the line at, e.g., the $\mathrm{K}_{1}$ minimum. This implies that one may use models with higher chromospheric minimum temperatures to fit the observed intensities, and this, in turn, has important implications for the empirical estimation of the amount of non-radiative energy deposition required to drive stellar chromospheres.

\section{Multidimensional Structures}

The solution of the transfer equation in the presence of inhomogeneities remains a central problem of the analysis of the solar and of stellar chromospheres. New methods for solving this difficult problem have been developed by Jones and Skumanich (1973) and by Jones (1973). Much further work in this area is needed to assess the reliability of the usual approaches of constructing multi-column (non-interacting columns) atmospheres, and for clarifying conceptually the physical significance (if any) of 'average' model atmospheres for stellar chromospheres and photospheres in which inhomogeneities are expected to exist.

\section{MODEL ATMOSPHERE COMPUTATIONS (PLANE-PARALLEL), GRIDS AND MODELS, SYNTHETIC SPECTRA}

\section{A. Continuous Opacity}

Enhancement of continuous absorption by $\mathrm{H}_{2}^{+}$due to non-LTE effects was proposed by Krishna Swamy and Stecher (1974) as an explanation of the 'missing' ultraviolet solar opacity. However a more complete study by Tarafdar and Vardya (in press, Astrophys. J. 1975) shows that $\mathrm{H}_{2}^{+}$is not a significant contributor to the solar U.V. opacity. Free-free opacities of 10 negative ions and 6 negative molecules have been computed by John (1975). A model atmosphere opacity code for the $\mathrm{CN}$ red system taking into account 470000 lines has been described by Carbon (1973). The differences in the location of $M$ dwarfs and $M$ giants in the (J-H, H-K) diagram have been attributed to $\mathrm{H}_{2} \mathrm{O}$ opacity in dwarfs by Glass (1975); Praderie and Stecher (1973) have shown that quasi-molecular $\mathrm{H}_{2}$ absorption obliterates the Silicon I $1520 \AA$ absorption edge in A stars.

\section{B. Blanketing}

An extensive tabulation of line blocking has been done by Ardeberg and Virdefors (1975) in the spectral range 3275 to $10000 \AA$ for stars of spectral type 05 to G0 and all luminosity classes. A useful discussion of the opacity probability distribution function technique has been given by Van Paradijs and Vardya (1975). Dragunova (1973) has evaluated the influence of blanketing effects in the atmospheres of $M$ stars on the $V$ and $R$ colors, Eberlein et al. (1973) have improved the computation of blanketing in the ultraviolet for early type atmospheres.

\section{Line Broadening}

Roueff (1975) has shown that exchange broadening with neutral hydrogen may be several times the Van den Waals broadening in the specific case of $\mathrm{Na} I$ lines. Foy (to be published in Astron. Astrophys.) has extended his study of the scatter found in the solar curves of growth to stellar atmospheres of much lower and much higher densities and shown that this scatter evolves as expected if attributed to a scatter on the damping constants of the lines. A main conclusion of this is that the use of an average curve of growth for the sun results in large systematic errors, spurious ionization ratios (Perrin et al., 1975) in differential analyses of a star much cooler than the sun or with a very different gravity or metal content with respect to the 
sun. The same warning applies to determinations of microturbulence in late type dwarfs, the ignorance of the actual scatter in damping producing artificially high velocities (Foy, 1976).

Empirical formulae for the broadening of iron lines by neutral hydrogen are given by Edmunds (1975).

\section{Grids of Models (LTE)}

An extensive grid of blanketed LTE models has been computed by Peytremann (1974a,b and $1975)$ in the range $\left(5000<T_{\text {eff }}<8500 \mathrm{~K}, 2 \leqslant \log \mathrm{g} \leqslant 4.5\right)$ for several metal/hydrogen ratios. Interpretation of broad-band photometric observations are given with the help of mono-. chromatic fluxes computed for those models. Similar models have been computed by Bell (1973). An extension to later spectral types for giants including $\mathrm{CN}$ and $\mathrm{CO}$ opacities has been computed by Gustafsson et al. (1975). Still later models have been computed for $\mathrm{K}$ and $\mathrm{M}$ dwarfs including $\mathrm{H}_{2} \mathrm{O}$, $\mathrm{CO}$ and $\mathrm{OH}$ absorbers by Kipper (1973). Wehrse (1973) has computed model atmospheres for white dwarfs.

\section{E. Non-LTE Model Atmospheres}

Models for extreme supergiants have been computed by de Jager and Neven (1975) and the fluxes predicted from $100 \AA$ to $6 \mu$. Deviations from LTE occur for $\tau<1$ but their effect is small on the continuum except in the far U.V. One of the authors (de Jager, 1975) infers from the position of supergiants with respect to the Eddington limit that the outward driving force due to radiation is approximately equal to the turbulent pressure gradients. Non-LTE effects in AOIa supergiants have also been investigated by Kudritzki (1974). Kudritzki (to be published in A stron. A strophys.) has computed non-LTE model atmospheres for subluminous $O$ stars.

Model chromospheres for F, G and K stars have been derived by Linsky and Ayres (1973), and Ayres, Linsky and Shine (1974) from non-LTE analyses of calibrated spectra of selected strong spectral lines (e.g., the Ca II H and K lines). Ayres, Linsky and Shine (1975) have suggested an explanation for the observed width-luminosity correlation of the Ca II K line (Wilson-Bappu effect) based on a non-LTE line formation theory. Frandsen (1974) has constructed a small grid of non-LTE model atmospheres for stars near spectral type A0.

\section{F. Non-LTE Abundance Analyses}

A review of effects of departures from LTE on stellar spectra has been given by Mihalas and Athay (1973). Specific abundance analyses for He I and Ne I in B stars have been performed by Auer and Mihalas (1973a, 1973b). This work showed that departures from LTE account fully for the discrepancies between the blue-violet and the red lines of He I found in LTE computations, and give a helium abundance of $\mathrm{N}(\mathrm{He}) /(\mathrm{H})=0.10$. In the neon spectrum, serious errors are made by an LTE computation: the non-LTE analysis yields an abundance of $\mathrm{N}(\mathrm{Ne}) /$ $\mathrm{N}(\mathrm{H})=10^{-4}$. This is down from the LTE abundance by a factor of five, and agrees with the cosmic abundance inferred from nebulae and the solar corona, and thus removes a long-standing discrepancy. Departures from LTE in the O I multiplet near $\lambda 7774 \AA$ in F and G stars have been analyzed by Johnson, Milkey and Ramsey (1974). Kelch (1975) has studied non-LTE effects on the $\mathrm{Na} I \mathrm{D}$ line formation in Arcturus.

\section{HYDRODYNAMICS IN STELLAR ATMOSPHERES, EXTENDED ATMOSPHERES, VELOCITY GRADIENTS, STELLAR WINDS}

Very rapid and important progress has been made recently in the study of extended and expanding atmospheres, and of stellar winds. Extensive reviews of this general area have been given by Böhm (1973) and by Hummer (1975). Significant progress has also been achieved in modeling the stochastic motions currently described as 'turbulence' and in solving the transfer problem in such a model. 


\section{A. Stochastic Motions in Stellar A tmospheres}

Worrall (1973) and Worrall and Wilson (1974) have questioned the reality of the so-called spectroscopic microturbulence in stellar atmospheres. From another side Hearn (1974) claimed that the energy requirement for maintaining microturbulence of the order of the accepted values for the sun and for Am stars was unacceptably high. Several groups have adopted a more rigorous approach for the representation of the velocity field using a stochastic model and computing the emergent spectrum averaged on all the realizations. This technique allows one to enter the finite size of the 'turbulent' elements into the picture and to replace the concepts of 'micro' and 'macro' turbulence by that of 'correlation length'. The Heidelberg group (Hundt, 1973, Gail et al., 1974; Traving, 1976; Gail, 1976 and others (Magnan, 1974, 1975; Schatzman and Magnan, 1975, H. and U. Frisch, 1975, 1976) have developed new, efficient techniques to solve the transfer equations in models with finite size elements. Gray $(1973,1975)$ has tried to distinguish between small-scale motions, random large-scale motions and rotation with Fourier transforms of observed line profiles. He concludes that small-scale motions are necessary to interpret the observations, and that the so-far unsuccessful attempts to distinguish between stellar rotation and large-scale motions are indeed solved, provided that the spectral resolution allows the second lobe of the Fourier transform of the rotational profile to be reached.

Glebocki (1973) has surveyed the microturbulence in 375 stars of spectral type $F, G, K$ and Reimers (1976) has given a review article on small- and large-scale motions in the Nice Colloquium 'Physics of motions in stellar atmospheres'. Smith (1973) has investigated the microturbulence in A stars using line profiles. Brooke et al. (1974) have demonstrated the sporadic character of large-scale motions in the atmosphere of red supergiants. In conclusion it seems that (1) some old values of the microturbulence on stellar atmospheres might be revised to lower values in light of improved line-formation theory, and of a better appreciation of the role of damping at the turn-off of the curve of growth (cf. line broadening section), but that (2) the energy in small scale modes is not going to disappear entirely as some too-enthusiastic astrophysicists have predicted.

\section{B. Extended Atmospheres}

Virtually all work on extended atmospheres assumes spherical geometry. New methods for solving transfer problems in spherical geometry have been given by Peraiah and Grant (1973) using a 'cell method' (i.e. a generalized discrete-ordinate method), and by Kunasz and Hummer (1974a), Mihalas and Hummer (1974b), and Schmid-Burgk (1975). These latter methods are based on direct ray-by-ray solutions (or the equivalent solution of the moment equations with variable Eddington factors) using either differential or integral-operator techniques. The array of techniques now available has sufficient generality and flexibility to allow us to consider the problem of radiative transfer in spherical geometry in astrophysical media to have been conclusively solved. Grids of static LTE spherical model atmospheres for early-type have been constructed by Castor (1974b) and Hundt $e$ t al. (1975). Non-LTE static spherical atmospheres for $O$ stars have been constructed by Mihalas and Hummer (1974b) and Kunasz, Hummer and Mihalas (1975). All of these calculations assume that the cause of the atmospheric extension is radiation forces acting on absorbing continua and lines, and use some kind of parameterization of these forces. Castor concludes that when an atmosphere becomes really extended, a strong stellar wind must occur and that static models become invalid. The effects of atmospheric extension upon intermediate-band colors has been discussed by Mihalas and Hummer (1974a, 1974b) and Mihalas (1975), and a comparison with observation has been effected by Morrison (1975). These analyses suggest that considerable atmospheric extension is present already at the depth of continuum formation, which provides constraints to be met by stellar wind models (see below). The transfer of radiation in dusty circumstellar envelopes has been studied by Apruzese (1975a,b) and by Schwartz (1975). Cassinelli and Hoffman (1975) have evaluated the polarization of light from extended atmospheres and its effect on interferometric measurements of angular sizes. Kunasz and Hummer (1974a) have presented a systematic study of line formation in spherical static media. 


\section{Expanding Atmospheres}

The solution of the line formation problem in expanding atmospheres presents considerable difficulty. The transfer equation in the observer's frame is easy to formulate, but difficult to solve. An observer's frame calculation has been carried out by Kunasz and Hummer (1975b) in a study of line-formation in expanding spherical atmospheres; in practice the expansion velocity must be quite modest (a few times the thermal velocity) for this method to be applied. It is far more attractive conceptually to work in the comoving frame of the fluid, but here the transfer equation is much more difficult to solve mathematically. Accurate formulations of the comoving frame transfer equation have been given by Castor (1972) and Haisch (1975). An integral-equation method for solving the comoving-frame equations was developed by Simonneau (1973) for the case of a linear velocity law. Noerdlinger and Rybicki (1974) have presented a differentialequation method for solving the comoving frame transfer equation in planar geometry; their approach is very general and flexible and may be applied for either complete redistribution or partial redistribution in the scattering process. Mihalas, Kunasz and Hummer (1974) have developed a general and flexible technique for solving the comovingframe equation in spherical geometry and applied it to parameterized problems of lineformation; subsequent studies of the picket-fence problem in expanding spherical media (Mihalas, Kunasz and Hummer, 1976a) and of the effects of terms of order v/c (Mihalas, Kunasz and Hummer, 1976b) have also been made. Kuan and Kuhi (1975) have studied line formation in P-Cygni stars using a Sobolev escape-probability technique; they conclude that P-Cygni stars have decelerating flows (in contrast to the trans-sonic flows observed in of and WR stars). Escape-probability techniques for both one-dimensional moving media have been developed by Magnan (1974a). Cannon and Cram (1974) have formulated a velocity-dependent source function for the case of time-dependent flow.

The effects of partial redistribution in the scattering process for line-formation in expanding media have been addressed by Vardavas (1974) and Cannon and Vardavas (1974) who use velocity-dependent redistribution functions which have been angle-averaged in the observer's frame. The inadequacies of such an approach have been emphasized by Magnan (1974b), and a more satisfactory calculation which performs the angle-averaging in the comoving frame has been given by Mihalas et al. (1976).

\section{Stellar Winds}

The theory of stellar winds for early-type stars has shown significant growth recently. Radiation-driven winds for Of and WR stars have received special attention. Cassinelli and Castor (1973) analyzed the effects for radiation forces and energy-input in optically thin winds. Castor (1974a) derived analytical approximations for the force exerted by radiation being absorbed in spectral lines in the presence of flow, and Castor, Abbott and Klein (1975) calculated self-consistent radiation-force-driven winds for Of stars. These models show a 'core-halo' structure, with a nearly static region where continuum formation occurs and a rapid rise in velocity to terminal velocity at some point higher in the atmosphere. The models predict the observed terminal velocities. In the region of continuum formation, the model is nearly planar, and has a steep density gradient. This predicts an absence of extension effects on continuum parameters, and may conflict with observation on this point. Cassinelli and Hartmann (1975) have constructed models in which the subsonic flow region is geometrically extended, and show that these models can fit observed energy distributions. Further work to clarify the actual structure in the trans-sonic region of the flow is needed. An alternative class of models has been developed by Hearn (1975) in which the wind is driven by a hot corona; Hearn $(1972,1973)$ has suggested mechanisms by which instabilities can be amplified in O-star atmospheres, and thus may provide the energy input required to heat a corona. Direct observational evidence for a corona around $\tau$ Sco has been presented by Rogerson and Lamers (1975). Truly satisfactory models for early-type winds will no doubt need to incorporate both mechanisms to some degree; it is clear that the radiation forces dominate the supersonic flow region, but the existence of a corona may prove crucial in the trans-sonic region. Mass-loss in Be 
stars has been studied by Limber (1974) who constructed steady-state models allowing for gravitational, pressure and magnetic forces. Marlborough and Zamir (1975) discuss the flow in rapidly rotating stars using an approach similar to that of Cassinelli and Castor (1973) to treat the coupling to the radiation field. The mass-loss problem in cool giant atmospheres has been studied by Salpeter (1974) and Kwok (1975) who analyzed the formation of dust grains, and the effects of radiation forces on the grains.

Considerable information concerning winds and coronae in later-type stars is beginning to emerge from ultraviolet observations. Gerola et al. (1974) have presented evidence for a corona around $\beta \mathrm{Gem}$. Asymmetries in ultraviolet line profiles, suggesting mass-loss in winds, have been observed in a number of stars by Moos et al. (1974), Kondo et al. (1975a,b), and Dupree (1975). These data indicate directly that winds occur over a wide range in the HR diagram and should provide valuable guidance to attempts to construct theoretical models of the flows.

\section{E. Dust Grains in Stellar Atmospheres}

Formation and subsequent effects of particles in stellar atmospheres have been investigated. Nucleation and grain growth have been studied by Salpeter $(1974 a, b)$ in the cool stellar atmospheres and by Noshida and Nakagawa (1973) in T Tauri envelopes. Jennings (1973) Salpeter (1974b), Maciel (1974), Fix and Alexander (1974), Gilman (1973) have studied the effect of grains on mass loss, and also Tabak et al. (1975).

\section{F. X-ray Production by Stellar Coronae}

Soft X-rays from Sirius have been reported by Mewe et al. (1975), Hills (1973) and Landini and Fossi (1973) have suggested that coronae of red giants may account for a large fraction of the soft X-ray galactic background. Negative results have been reported by Margon, Manson and Sanford (1974) from Copernicus observations and negative results more stringent by one order of magnitude have been reported by Crudacce et al. (1975) for the two nearby objects Arcturus and Procyon. If the coronae of these two stars are typical, galactic soft X-rays do not originate mainly in stellar coronae.

\section{G. Stellar Atmospheres as Transition Zone between Stellar Interiors and Interstellar Medium}

Pecker et al. (1973) and Thomas (1973) have developed general physical considerations on the significance of mass flux in stellar atmospheres and on the role of atmospheres as a transition zone between stellar interiors (where non-thermal kinetic energy-storage takes place) and the interstellar medium which is far from thermodynamic equilibrium. Dumont et al. (1973) have attempted to explain the $\mathrm{H} \alpha$ emission on T Tauri stars by a chromosphere, as opposed to an extended envelope mechanism. A second paper on $\mathrm{T}$ Tauri emission lines by Thomas and Heidmann is in press as well as a paper by Cannon and Thomas on the origin of stellar winds (to be published in $A p J$ 1976).

\section{DIFFUSION PROCESSES}

Michaud et al. (1974) have suggested that the mercury isotope anomaly in manganese stars can be explained by diffusion processes. Wolff and Wolff (1974) in a systematic study of manganese stars have found strong arguments supporting Michaud's view. Michaud and Montmerle (to be published in Astrophys. J. Suppl. 1976) have written an extensive review of the theoretical treatment of diffusion processes and have pointed out that in a helium rich atmosphere, hydrogen is accelerated outwards by the electrical field generated by the slight separation between electrons and ions. Vauclair et al. $(1974 \mathrm{a}, \mathrm{b})$ have shown that in A stars gravitational sinking of helium can destroy the second ionization and therefore increase the importance of other diffusion processes by decreasing the size of the mixed zone at the 
surface of the stars (limited then to the hydrogen convective zone). Another interesting effect of diffusion is the depletion of lithium, beryllium and boron in the solar envelope by diffusion of these elements towards the inner region where they are thermally destroyed (Schatzman and Vauclair, 1973). Havnes (1975) has studied the horizontal diffusion in peculiar stars with spotted chemical composition. He finds that the diffusion time is shorter than the life time of the star and that periodic variations of lines should then damp with the age of the stars.

At the Vienna Colloquium on Ap stars Michaud presented a very up to date review of diffusion processes. Vauclair (1975) gives a possible explanation of both helium enhancement and depletion in combining diffusion processes with mass loss. Shore and Adelman (1974) have discussed diffusion processes in presence of a magnetic field. Vauclair has given a synthetic review of the alternative diffusion processes by meridional circulation and turbulence at the IAU Vienna Colloquium No. 32.

\section{MAGNETISM IN STELLAR ATMOSPHERES}

Thermal convective instability in the presence of a magnetic field has been studied by several authors. Singla and Talwar (1973) have considered the case of an isothermal atmosphere. Sharma (1974a, b) and Sharma and Prakasch (1974) have concluded that the instability criterion is unaffected by a vertical magnetic field. Staude (1973) found that the stratification is unaffected at optical depths larger than one but not at smaller optical depths in a sunspot-like magnetic field. The oblique rotator model for magnetic $A$ stars has been found satisfactory by a few authors (Stift, 1975; Mégessier, 1975) and unsatisfactory by others (Scholz, 1975). Sidlichovsky (1974) and House and Steinitz (1975) have formulated the non-LTE transfer equations for line formation in the presence of a magnetic field. Several authors have studied Be star envelopes with a magnetic field (Morozov, 1973a,b; Saito, 1974; Okamoto, 1974). Transfer of rotational momentum in the envelope through the magnetic field could be an important factor.

\section{THE SOLAR ATMOSPHERE}

A leading discovery in the physics of the solar atmosphere is the emergence of an understanding of the so-called five minute solar oscillations. Several theoretical works (Wolff (1972), Ulrich (1970) Ando and Osaki (1975)) have computed the non-radial high horizontal modes of vibration of the solar envelope as well as their stability. It tums out that horizontal modes with quantum numbers 1 of the order of one hundred and radial numbers from two to four or five are unstable. At a given value of the horizontal wave number $\mathbf{k}_{\mathbf{x}}$ one should then find discrete time frequencies corresponding to the proper values of the radial modes. Deubner (1975) has been able to demonstrate beautifully the existence of such a structure. If the instability region is roughly centred at a $300 \mathrm{~s}$ period, the actual situation is far more complex, three to four periods showing up at a given value of $k_{x}$. The lowest radial mode is generally stable but the oscillation of period above $300 \mathrm{~s}$ formerly reported by Frazier can be understood as a $\mathrm{p}_{1}$ mode with Deubner's diagramme. The oscillation is still detectable at very small values of 1 as observationally shown by Fossat (1974). Potential implications explaining motions in other stellar atmospheres are very large.

Departures from LTE in solar type stars have been theoretically investigated by Lites and Cowley (1974). Smith (1974) has compared the solar spectrum to the Lites and Cowley predictions and found that the actual departures are negligible for high excitation lines, and are about one-half of those predicted by these authors for strong, low excitation lines.

The U.V. and far U.V. solar spectrum display several interesting new features: Jordan (1975) finds that the intensities of $\mathrm{He} \mathrm{I}$, He II lines are anomalously strong, and can be explained if the lines are excited by electrons at a temperature higher than that of ionization equilibrium. Boland et al. (1975) find that line profiles in the range $1400 \AA<\lambda<2200 \AA$ imply a non-thermal velocity broadening with subsonic speeds of the order of $10^{\circ} \mathrm{K}$. Possible wave modes for mechanical energy flux to the corona are considered. 
Gehren (1975) has been able to account for the Na I D line profiles by a non-LTE treatment of the problem, in conjunction with an anisotropic and depth-variable model for the random velocity field.

Mount and Linsky (1975a,b) have given a revised logarithmic carbon abundance in the solar atmosphere equal to $8.35 \pm 0.15$ in the usual scale $\log \mathrm{H}=12.0$.

Mason (1975) has improved the interpretation of Fe X, XI, XIV and Ca XII, XIII and XV coronal lines and deprived an iron abundance of 7.85 and a calcium abundance of 6.8 .

\section{ABUNDANCES AND ATMOSPHERE PARAMETERS IN INDIVIDUAL OBJECTS}

A general catalogue of atmospheric parameters and iron/hydrogen ratios is published by Morel et al. in the proceedings of the IAU Symposium No. 72.

\section{A. Early-type Stars}

Code, Davis Bless and Hanbury Brown (1976) have combined angular diameter measurements of 32 bright early stars (O5 $\mathrm{f}$ to $\mathrm{F} 8$ ) with ground based and $\mathrm{OAO}-2$ absolute flux measurements to derive effective temperatures and bolometric corrections. Rogerson and Lamers $(1975,1976)$ have identified broad lines of OVI and NV in $\tau$ Scorpii on Copernicus observations; these imply an extremely high outward velocity of the matter as well as temperatures characteristic of a corona. Lamers and Snijders (1975) have discussed space observations of the Mg II lines in early type stars. Heap (1975) has interpreted the complex spectrum of the shell star $\zeta$ Tauri in the range 1100-2050 $\AA$ and found abundance deficiencies in carbon and silicon. Underhill (1975) has discussed the UV spectrum of $B$ supergiants in general and that of $\eta \mathrm{CMa}$ in particular. Nissen (1974) has determined the helium to hydrogen ratio from narrow band photoelectric observations in 148 main sequence B stars. Lyubinkov (1974) has proposed a fast method for evaluating the helium abundance in hot stars based on a comparison of He $1 \lambda 4471$ to $\mathrm{H} \gamma$. Van der Hucht (1975) has found an usually great contribution of the Wolf Rayet component of $\gamma^{2}$ Velorum in the $U V$ at $2000 \AA$ which is attributed to a high helium abundance. Adelman (1973) has determined the chemical composition of the $B_{9} V$ star $\nu$ Capricorni.

\section{B. Helium-rich and Hydrogen-deficient Stars}

A very large amount of work has been devoted to helium-rich stars these last three years. A nice review of their general properties is given by Osmer and Petersson (1974) who also give a grid of models for relevant $\mathrm{He} / \mathrm{H}$ ratios. In brief they form a well-defined group with the following characteristics: (1) $\mathrm{He} / \mathrm{H} \simeq 1$, (2) effective temperatures near $\mathrm{B} 0-\mathrm{B} 2$, (3) main sequence gravity, (4) slow rotation, (5) $\mathrm{O} / \mathrm{H}$ and $\mathrm{N} / \mathrm{H}$ ten times larger than solar values, (6) normal abundance of $\mathrm{C}, \mathrm{Si}, \mathrm{Mg}, \mathrm{Al}$. Among others the following objects have been analysed: HD 60344 by Kaufmann and Hunger (1975), HD 184927 by Higginbetham and Lee (1974), HD 37776 by Clas-Offick (in press). Hydrogen-poor stars have also raised much interest. Popper's star HD 124448 has been analysed by Schönberner and Wolf (1973) and found to be made of $99 \%$ helium, $0.8 \%$ carbor and $0.2 \%$ nitrogen. Hunger and Kaufmann (1973) have analyzed HD 144941 and BD $-69^{\circ} 2698$; Kaufmann and Schönberner (1976) BD $-9^{\circ} 4395$, and Schönberner (1973) HD 135 485. Dufton (1973) has found an early-type metal rich star: HD 135485.

\section{Ap, Am and A Stars}

The 32nd IAU Colloquium (Vienna, 1975) gives valuable up-to-date information on the atmospheres of these objects. Already mentioned is the considerable ground gained by the diffusion process over other types of explanation for the peculiar abundances in Ap or Am stars. (See Stickland's (1975) review paper for example.) Little (1974) has found somewhat new peculiarities in the object HD 168733. Zverko (1974) (1975) has computed a model atmosphere 
for 53 Aur. Khoklova and Rjabchikov (1975) have produced a spotted model for the strontium star HD 140160 with the overabundances of Sr by a factor of $10^{3}$ in 3 spots. Boesgaard et al. (1974) have determined the boron abundance in Vega from the resonance line of B II $\lambda 1362 \AA$ and derived $\mathrm{a} B / \mathrm{Be}$ ratio of 10 .

Fracassini et al. (1975) have determined the apparent radii of 116 A5-F5 dwarfs of the catalogue of the Geneva Observatory.

\section{Solar-type Stars}

The spectrum of Arcturus has been studied now with almost as much care as the solar spectrum thanks to Griffin's atlas (1968). Mäckle et al. (1975a, b) have performed a detailed analysis of the object and have found a surprisingly low gravity $(\log g=0.9 \pm 0.35)$ and metal deficiencies higher than formerly accepted: running from a factor of 5 for the iron group to a factor of 10 for heavy elements. Lithium was found much depleted with respect to the sun. Blackwell et al. (1975) find from the continuous flux distribution of Arcturus an effective temperature $250 \mathrm{~K}$ higher than Mäckle et al.

Many stars of type $F, G$ and $K$ have been analysed for population-study purposes. The case for 'super-metal-rich' stars has been further weakened by Griffin's (1975) analysis of a red giant in $\mathrm{M} 67$ with the result of $[\mathrm{Fe} / \mathrm{H}]=-0.3$ relative to the sun. But Gustaffson et al. (1974) using a very narrow band photometry find again some support for a metal-content much greater than that of the Hyades in some 80 giants with an echelle spectrometer.

Hearnshaw $(1973,1974,1975)$ has studied the carbon and iron abundance in thirty $F$ and $G$ type stars in the northern hemisphere, and 11 southern stars. Cayrel de Strobel and Pasinetti (1975) find that the high-velocity giant HD 6497 is not metal-poor. The authors discuss this result which appears discrepant with the membership of the stars in Eggen's Arcturus group. Helfer and Jennens $(1975)$ have determined $[\mathrm{Fe} / \mathrm{H}]$ in $\mathrm{K}$ giants at $10 \mathrm{kpc}$ near the galactic plane. They do not find any significant difference with nearby K giants. Da Silva (1975) has studied 3 objects HR 3018, HD 91324 and $\alpha \mathrm{Crv}$ and has drawn conclusions about the age-metal content correlation in the solar neighbourhood. F. and M. Spite (1975) have found the cool halo star HD 128279 metal-poor by a factor one hundred with respect to the sun. Williams (1973) finds $\epsilon$ Leonis to have the chemical composition of the sun in spite of its photometric properties. Perrin (1975), (see also ref. in section 4C) has obtained the chemical composition of $12 \mathrm{G}$ and K stars.

\section{E. Very Late-type Stars}

Boyarchuk has given a review on the chemical composition of the late-type stars at the IAU Symposium No. 66.

The isotopic ratio ${ }^{12} \mathrm{C} /{ }^{13} \mathrm{C}$ still raises considerable interest as one of the main tracer of stellar evolution. Thompson (1973) has pointed out the problems that are encountered when using the frequently-saturated first-harmonic bands of $\mathrm{CO}$ for determining this ratio. To circumvent this difficulty, the 2-0 and 4-0 red systems have been used by Tomkin and Lambert (1974) for getting ${ }^{12^{2}} \mathrm{C} /{ }^{13} \mathrm{C}$ in $\alpha$ Tau, $\beta$ Gem and $\mu$ Leo, by Lambert $e$ t al. (1974) for obtaining ${ }^{12} \mathrm{C} /{ }^{13} \mathrm{C}$ in $\alpha$ Orionis and by Lambert and Tomkin in $\epsilon$ Peg and Lambert and Sneden in $\alpha$ Boo and $\alpha$ Ser. All these ratios range between 5 and 18 times larger than the solar system values. Thompson and Johnson (1974) obtain a lower limit of 20 in $\alpha$-Herculis. Bell and Dickens (1974) have obtained ${ }^{12} \mathrm{C} /{ }^{13} \mathrm{C}=10$ in the $\mathrm{CH}$ star of Omega Centauri. Williams (1975) has determined the barium abundance in 200 evolved stars by narrow band photometry and spectral synthesis. Lee (1974) has analysed the CH star HD 198269 and found a very high $\mathrm{C} / \mathrm{Fe}$ ratio of nearly one hundred and $(\mathrm{Fe} / \mathrm{H})=-1.5$.

Boyarchuk (1972a, b) has studied the abundance of lithium in two M5 dwarfs and in SR variable M stars. The Querci's $(1974,1975 \mathrm{a}, \mathrm{b})$ have further studied carbon stars with model atmospheres using opacity probability distribution functions. They tried to find evidence in favor of either the $3 \alpha$ processes or the CNO bicycle in UU Aur and in YCVn. Uus (1974) has looked for evolutionary abundance changes in the envelopes of moderate mass red supergiants. 
Fay and Johnson (1973) have studied Betelgeuse with a model atmosphere. They find $[\mathrm{C} / \mathrm{H}]=-1.0$ with respect to the sun and obtain a $\mathrm{C} / \mathrm{O}$ ratio smaller in Betelgeuse than in the sun. Shavrina and Yakovina (1973) find that $\alpha$ Sco and $\alpha$ Her A have a chemical composition similar to the sun. Shavrina (1974) has also determined the abundance of Ti $O$ and $O$ in the 5 stars $\alpha$ Sco, $\gamma^{2}$ Lyra, $\alpha$ Her A, $\epsilon$ Peg.

Bues (1973) has determined abundances in white dwarfs with carbon bands.

N.B. An important list of references of works done in U.S.S.R. reached the President too late to be included in this report. An addendum has been prepared and distributed to the members of the Commission. It is available on request.

\section{BIBLIOGRAPHY}

\section{General References}

\section{Books}

Bappu, M. K. V. and Sahade, J., (eds): 1973, Wolf-Rayet and High Temperature Stars, IAU Symp. 49, Reidel, Dordrecht.

Baschek, B., Kegel, W. H. and Traving, G. (eds.): 1975, Problems in Stellar Atmospheres and Envelopes, Springer-Verlag, Berlin.

Cayrel, R. and Steinberg, M. (eds.): 1976, Physique des Mouvements dans les Atmosphères Stellaires, CNRS, Paris.

Hauck, B. (ed.): 1976, Abundance Effects in Classification, IAU Symp. 73, Reidel, Dordrecht.

Ivanov, V.V. (transl. and ed. by D. G. Hummer): 1973, Transfer of Radiation in Spectral Lines, NBS Special Publication 385, US Government Printing Office, Washington.

Jordan, S. D. and Avrett, E. H.: 1973, Stellar Chromospheres, NASA Publication SP-317, US Government Printing Office, Washington.

Morel et al.: 1975, Catalogue of Atmospheric Parameters and [Fe/H] Determinations, IAU Symp. 73, Reidel, Dordrecht.

Novotny, E. C.: 1973, Introduction to Stellar Atmospheres and Interiors, Oxford Univ. Press, New York.

Review articles

Altizer, R. J.; 1973, Quart. J. Roy. Astron. Soc. 14, 318.

Blackwell, D. E.: 1974, Quart. J. Roy. Astron. Soc. $15,224$.

Gray, D. F. and Evans, J. C.: 1973, Roy. Astron. Soc. Can. J. 67, 241.

Mihalas, D.: 1973, Publ. Astron. Soc. Pacific 85, 593.

Mihalas, D. and Athay, R. G.: 1973, Ann. Rev. Astron. Astrophys. 11, 187.

Myerscough, V. P. and Peach, G.: 1973, Case Studies in Atomic Physics 2, 245.

Pagel, B. E. J.: 1973, Space Sci. Rev. 15, 1.

Preston, G. W.: 1974, Ann. Rev. Astron. Astrophys. 12, 257.

Wallerstein, G.: 1973, Ann. Rev. Astron. Astrophys. 11, 115.

\section{References (by sections of the report)}

3. Radiative Transfer (Plane-Parallel)

\section{A. The Non-LTE Transfer Equation}

Auer, L. H. and Heasly, J. N.: 1976, Astrophys. J. 205, 165.

Kalkofen, W.: 1974, Astrophys. J. 188, 105.

\section{B. Partial Redistribution Effects on Line Formation}

Heasley, J. N. and Kneer, F.: 1975, submitted to Astrophys. J.

Milkey, R. W. Ayres, T. R., and Shine, R. A.: 1975, Astrophys. J. 197, 143. 
Milkey, R. W. and Mihalas, D.: 1973a, Astrophys. J. 185, 709.

Milkey, R. W. and Mihalas, D.: 1973b, Solar Phys. 32, 361 .

Milkey, R. W. and Mihalas, D.: 1974, Astrophys. J. 192, 769.

Milkey, R. W., Shine, R. A., and Mihalas, D. 1975a, Astrophys. J. 199, 718

Milkey, R W., Shine, R. A., and Mihalas, D.: 1975b, Astrophys. J. 202.

Shine, R. A., Milkey, R. W., and Mihalas, D.: 1975a, Astrophys. J. 199, 724.

Shine, R. A., Milkey, R. W., and Mihalas, D.: 1975b, Astrophys. J. 201.

Vardavas, I. M. and Cram, L. E.: 1974, Solar Phys. 38, 367.

Vernazza, J. E., Avrett, E. H., and Loeser, R.: 1973, Astrophys. J. 184, 605.

\section{Multidimensional Structures}

Jones, H. P.: 1973, Astrophys. J. 185, 183.

Jones, H. P. and Skumanich, A.: 1973, Astrophys. J. 185, 167.

\section{Model Atmosphere Computations}

\section{A. Continuous Opacity}

Carbon, D. F.: 1973, Astrophys. J. 183, 903.

Glass, I. S.: 1975, Monthly Notices Roy. Astron. Soc. 171, 19.

John, T. L.: 1975, Monthly Notices Roy. Astron. Soc. 170, 5.

Krishna Swamy, K. S. and Stecher, T. P.: 1974, Astrophys. J. Letters 194, L153.

Praderie, F. and Stecher, T. P.: 1973, Astron. Astrophys. 23, 49.

Tarafdar, S. P. and Vardya, M. S.: 1975, Astrophys. J. (in press).

\section{B. Blanketing}

Ardeberg, A. and Virdefors, B.: 1975, Astron. Astrophys. 39, 21.

Dragunova, A. V.: 1973, Astron. Tsirk. No. 750, 1.

Eberlein, D., Scholz, M., and Traving, G.: 1973, Astron. and Astrophys. 24, 295.

Paradijs, J. van and Vardya, M. S.: 1975, Astrophys. Space Sci. 33, L9.

\section{Line Broadening}

Edmunds, M. G.: 1975, Astron. Astrophys. 38, 137.

Perrin, M. N., Cayrel, R., and Cayrel de Strobel, G.: 1975, Astron. Astrophys. 39, 97.

Roueff, E.: 1975, Astron. Astrophys. 38, 41.

Foy, R.: 1976, 250ème colloque international du CNRS, Physique des mouvements dans les atmosphères stellaires, Nice.

\section{Grids of Models}

Bell, R. A.: 1973, Monthly Notices Roy. Astron. Soc. 164, 197.

Gustafsson, B., Bell, R. A., Eriksson, K., and Norlund, A.: 1975, Astron. Astrophys. 42, 407.

Kipper, T.: 1973,Publ. Tartu Astrophys. Obs. 41, 62.

Pey tremann, E.: 1974, Astron. Astrophys. 33, 203.

Pey tremann, E.: 1974, Astron. Astrophys. Suppl. 18, 81.

Pey tremann, E.: 1975, Astron. Astrophys. 38, 417.

Wehrse, R.: 1973, Mitt. Astron. Ges. 32, 232.

\section{E. Non-LTE Model Atmospheres}

Ayres, T. R., Linsky, J. L., and Shine, R. A.: 1974, Astrophys. J. 192, 93.

Ayres, T. R., Linsky, J. L., and Shine,R. A.: 1975, Astrophys. J. Letters 195, L121.

Frandsen, S.: 1974, Astron. Astrophys.37, 139.

Jager, C. de: 1975, Phil. Trans. Roy. Soc. London A 279, 421.

Jager, C. de and Neven, L.: 1975, Astrophys. Space Sci. 33, 295.

Kudritzki, R. P.: 1973, Astron. Astrophys. 28, 103. 
Kudritzki, R. P.: 1975, Astron. Astrophys. (in press).

Linsky, J. L. and Ayres, T. R.: 1973, Astrophys. J. 180, 473.

\section{F. Non-LTE Abundance Analyses}

Auer, L. H. and Mihalas, D.: 1973a, Astrophys. J. Suppl. 25, 433.

Auer, L. H. and Mihalas, D.: 1973b, Astrophys. J. 184, 151.

Johnson, H. R., Milkey, R. W., and Ramsey, L. W. 1974, Astrophys. J. 187, 147.

Kelch, W. L.: 1975, Astrophys. J. 195, 679.

Mihalas, D. and Athay, R. G.: 1973, Ann. Rev. Astron. Astrophys. 11, 187.

\section{Hydrodynamics in Stellar A tmospheres}

Böhm, K.-H.: 1973, IAU Symp. 51, 148.

Hummer, D. G.: 1975, IAU Symp. 70 (in press).

\section{A. Stochastic Motions in Stellar Atmospheres}

Brooke, A. L., Lambert, D. L., and Barnes, T. G. III: 1974, Publ. Astron. Soc. Pacific 86, 419.

Frisch, H.: 1975, Astron. Astrophys. 40, 267.

Frisch, H.: 1976, 250ème colloque international du CNRS, Phy sique des mouvements dans les atmosphères stellaires, Nice.

Frisch, U.: 1976, 250ème colloque international du CNRS, Physique des mouvements dans les atmosphères stellaires, Nice.

Gail, H. P., Hundt, E., Kegel, W. H., Schmid-Burgk, J. and Traving, G.: 1974, Astron. Astrophys. 32, 65.

Gail, H.P.: 1976, 250ème colloque international CNRS, Physique des mouvements dans les atmosphères stellaires, Nice.

Glebocki, R.: 1973, Acta Astron. 23, 135.

Gray, D. F.: 1973, Astrophys. J. 184, 461.

Gray, D. F.: 1975, Astrophys. J. 202, 148.

Hearn, A. G.: 1974, Astron. Astrophys. 31, 415.

Hundt, E.: 1973, Astron. Astrophys. 29, 17.

Magnan, C.: 1974, J. Quant. Spectroscopic Radiative Transfer 14, 123.

Magnan, C.: 1974, Astron. Astrophys. 33, 139.

Magnan, C.: 1975, J. Quant. Spectroscopic Radiative Transfer 15, 979.

Reimers, D.: 1976, 250ème colloque international du CNRS, Physique des mouvements dans les atmosphères stellaires, Nice.

Schatzman, C. and Magnan, C.: 1975, Astron. Astrophys. 38, 373.

Smith, M. A.: 1973, Astrophys. J. 182, 159.

Traving, C.: 1976, 250ème colloque international du CNRS, Physique des mouvements dans les atmosphères stellaires, Nice.

Worral, G.: 1973, Nature, Phys. Sci. 241, 7.

Worrall, G. and Wilson, A. M.: 1974, Vistas Astron. 15, 39.

\section{B. Extended Atmospheres}

Apruzese, J. P.: 1975a, Astrophys. J. 196, 753.

Apruzese, J. P.: 1975b, Astrophys. J. 196, 761.

Cassinelli, J. P. and Hoffman, N. M.: 1975, submitted to Astrophys. J.

Castor, J. I.: 1974b, Astrophys. J. 189, 273.

Hundt, E., Kodaira, K., Schmid-Burgk, J. and Scholz, M.: 1975, Astron. Astrophys. 41, 37.

Kunasz, P. B. and Hummer, D. G.: 1974a, Monthly Notices Roy. Astron. Soc. 155, 19.

Kunasz, P. B., Hummer, D. G. and Mihalas, D.: 1975, Astrophys. J. 202.

Mihalas, D.: 1975, in Phillip, A. G. D. and Hayes, D. S. (eds.): Multicolor Photometry and the Theoretical

HR Diagram Obs., Dudley, Albany, p. 241.

Mihalas, D. and Hummer, D. G.: 1974a, Astrophys. J. Letters 189, L39.

Mihalas, D. and Hummer, D. G.: 1974b, Astrophys. J. Supp. 28, 343.

Morrison, N. D.: 1975, Astrophys. J. 200, 113.

Periah, A. and Grant, I. P.: 1973, J. Inst. Math. Appl. 12,75.

Schmid-Burgk, J.: 1975, Astron. Astrophys. 40, 249.

Schwartz, R. D.: 1975, Astrophys. J. 196, 745. 


\section{Expanding Atmospheres}

Cannon, C. J. and Cram, L. E.: 1974, J. Quant. Spectrosc. Radiative Transfer 14, 93.

Cannon, C. J. and Vardavas, I. M.: 1974, Astron. Astrophys. 32, 85.

Castor, J. I.: 1972, Astrophys. J. 178, 779.

Haisch, B. M.: 1976, Astrophys. J. (in press).

Kuan, P. and Kuhi, L. V.: 1975, Astrophys. J. 199, 148.

Kunasz, P. B. and Hummer, D. G.: 1974b, Monthly Notices Roy. Astron. Soc. 166, 57.

Magnan, C.: 1974a, J. Quant. Spectrosc. Radiative Transfer 14, 123.

Magnan, C,: 1974b, Astron. Astrophys. 35, 233.

Mihalas, D., Kunasz, P. B. and Hummer, D. G.: 1975, Astrophys. J. 202, 92.

Mihalas, D., Kunasz, P. B. and Hummer, D. G.: 1976a, Astrophys. J. 203, 647.

Mihalas, D., Kunasz, P. B. and Hummer, D. G.: 1976b, Astrophys. J. (in press).

Mihalas, D., Shine R. A., Kunasz, P. B., and Hummer, D. G.: 1976, Astrophys. J. (in press).

Noerdlinger, P. D. and Rybicki, G. B.: 1974, Astrophys. J. 193, 651.

Simmoneau, E.: 1973, Astron. Astrophys. 29, 357.

Vardavas, I. M.: 1974, J. Quant. Spectrosc. Radiative Transfer 14, 909.

\section{Stellar Winds}

Cassinelli, J. P. and Castor, J. I.: 1973, Astrophys. J. 179, 189.

Cassinelli, J. P. and Hartmann, L.: 1975, Astrophys. J. (in press).

Castor, J. I.: 1974a, Monthly Notices Roy. Astron. Soc. 169, 279.

Castor, J. I., Abbott, D. C. and Klein, R. I.: 1975, Astrophys. J. 195, 157.

Dupree, A. K.: 1975, Astrophys. J. Letters 200, L27.

Gerola, H., Linsky, J. L., Shine, R., McClintock, R., Henry, R. C. and Moos, H. W.: 1974, Astrophys. J.

Letters 193, L107.

Hearn, A. G.: 1972, Astron. Astrophys. 19, 417.

Hearn, A. G.: 1973, Astron. Astrophys. 23, 97.

Hearn, A. G.: 1975, Astron. Astrophys. 40, 355.

Kondo, Y., Morgan, T. H., and Modisette, J. L.: 1975a, Astrophys. J. Letters 196, L125.

Kondo, Y., Morgan, T. H., and Modisette, J. L.: 1975b, Astrophys. J. Letters 198, L37.

Kwok, S.: 1975, Astrophys. J. 198, 583.

Limber, D. N.: 1974, Astrophys. J. 192, 429.

Malborough, J. M. and Zamir, M.: 1975, Astrophys. J. 195, 145.

Moos, H. W., Linskey, J. L., Henry, R. C., and MoClintock, W.: 1974, Astrophys. J. Letters 88, 193.

Rogerson, J. B. and Lamers, H.: 1975, Nature (in press).

Salpeter, E.; E.: 1974, Astrophys. J. 193, 585.

\section{E. Dust Grains in Stellar Atmospheres}

Fix, J. D. and Alexander, D. R.: 1974, Astrophys. J. Letters 188, L91.

Gilman, R. C.: 1973, Monthly Notices Roy. Astron. Soc. 161, 3 P.

Jennings, M. C.: 1973, Astrophys. J. 185, 197.

Maciel, W. J.: 1974, Astrophys. Letters 15, 177.

Noshida, S. and Nakagawa, K.: 1973, Prog. Theor. Phys. 49, 1152.

Salpeter, E. E.: 1974a, Astrophys. J. $193,579$.

Salpeter, E. E.: 1974b, Astrophys. J. 193, 585.

Tabak, R. G., Hirth, J. P., Meyrick, G., and Roark, T. P.: 1974, Astrophys. J. 196, 457.

\section{F. X-ray Production by Stellar Coronae}

Crudacce, R., Bowyer, S., Malina, R., Margon, B., and Lampton, M.: 1975, Astrophys. J. Letters 202, L9.

Hills, J. G.: 1973, Astrophys. J. Letters 14, 69.

Landini, M. and Fossi, B. C.: 1973, Astron. Astrophys. 25, 9.

Margon, B., Mason, K. O., and Sanford, P. W.: 1974, Astrophys. J. Letters 194, L75.

Mewe, R., Heise, J., Gronenschild, E. H. B. M., Brinkman, A. C., Schrijver, J., and Den Boggende, A. J. F.: 1975, Nature 256, 711 . 


\section{G. Stellar atmospheres as transition zone between stellar interiors and interstellar medium}

Dumont, S., Heidmann, N., Kuhi, L. V., and Thomas, R. N.: 1973, Astron. Astrophys. 29, 199.

Pecker, J. C., Praderie, F., and Thomas, R. N.: 1973, Astron. Astrophys. 29, 289.

Thomas, R. N.: 1973, Astron. Astrophys. 29, 297.

\section{Diffusion Processes}

Kobay ashi, M. and Osaki, Y.: 1973, Publ. Astron. Soc. Japan 25, 495.

Michaud, G., Reeves, H., and Charland, Y.: 1974, Astron. Astrophys. 37, 313.

Michaud, G., Charland, Y., Vauclair, S., and Vauclair, G.: 1975, preprint.

Shore, S. N. and Adelman, S. J.: 1974, Astrophys. J. 191, 165.

Havnes, O.: 1975, Astron. Astrophys. 38, 105.

Michaud, G.: 1973, Astrophys. J. Letters 15, 143.

Schatzman, E. and Vauclair, S.: 1973, Comments Astrophys. Space Phys. 5, 159.

Vauclair, S.: 1975, Astron. Astrophys. (to be published).

Vauclair, G., Vauclair, S. and Pamjatnikh, A.: 1974a, Astron. Astrophys. 31, 63.

Vauclair, S., Michaud, G. and Charland, Y.: 1974b, Astron. Astrophys. 31, 381.

Wolff, S. C. and Wolff, R. J.: 1974, Astrophys. J. 194, 65.

\section{Magnetism in Stellar Atmospheres}

House, L. L. and Steinitz, R.: 1975, Astrophys. J. 195, 235.

Megessier, C.: 1975, Astron. Astrophys. 39, 263.

Morozov, V. N.: 1973a, Astrofiz. 9, 387.

Morozov, V. N.: $1973 \mathrm{~b}$, Astrofiz. 9, 567.

Okamoto, I.: 1974, Monthly Notices Roy. Astron. Soc. 166, 683.

Saito, M.: 1974, Publ. Astron. Soc. Japan 26, 103.

Scholz, G.: 1975, Astron. Nachr. 296, 31.

Sharma, R. C.: 1974a, Z. Naturforsch. 29a, 867.

Sharma, R. C.: 1974b, Astron. Astrophys. 32, 223.

Sharma, R. C. and Prakash, K.: 1974, Astrophys. Space Sci. 31, 43.

Sidlichovsky, M.: 1974, Bull. Astron. Inst. Czech. 25, 198.

Singla, M. and Talwar, S. P.: 1973, Astron. Astrophys. $24,441$.

Staude, J.: 1973, Astron. Nachr. 294, 113.

Stift, M. J.: 1975, Monthly Notices Roy. Astron. Soc. 172, 133.

\section{The Solar A tmosphere}

Ando, H. and Osaki, Y.: 1975,Publ. Astron. Soc. Japan 27, 581.

Boland, B. C., Dyer, E. P., Firth, J. G., Gabriel, A. H., Jones, B. B., Jordan, C., Mowhirter, R. W. P., Monk, P., and Turner, R. F.: 1975, Monthly Notices Roy. Astron. Soc. 171, 697.

Deubner, F. L.: 1975, Astron. Astrophys. 44, 371.

Fossat, E.: 1974, Astrophys. J. 193, L97.

Gehren, T.: 1975, Astron. Astrophys. 38, 289.

Jordan, C.: 1975, Monthly Notices Ruy. Astron. Soc. 170, 429.

Lites, B. W. and Cowley, C. R.: 1974, Astron. Astrophys. 31, 361.

Mason, H. E.: 1975, Monthly Notices Roy. Astron. Soc. 170, 651.

Mason, H. E.: 1975, Monthly Notices Roy. Astron. Soc. 171, 119.

Mount, G. H. and Linsky, J. L.: 1975, Astrophys. J. Letters 202, L51.

Smith, M. A.: 1974, Astrophys. J. 190, 481.

Ulrich, R. K.: 1970, Astrophys. J. 162,993.

Wolff, C. L.: 1972, Astrophys. J. Letters 177, L87.

9. Abundances and A tmospheric Parameters in Individual Objects

\section{A. Early type Stars}

Adelman, S. J.: 1973, Astrophys. J. 182, 531.

Code, A. D., Davis, J., Bless, R. C., and Hanbury Brown, R.: 1976, Astrophys. J. (in press). 
Heap, S. R.: 1975, Phil. Trans. Roy. Soc. London A279, 371.

Hucht, K. A. van der: 1975, Phil. Trans. Roy. Soc. London A279, 451.

Lamers, M. J. G. L. M. and Snijders, M. A. J.: 1975, Astron. Astrophys. 41, 259.

Lyvbimkov, L. S.: 1974, lzv. Krymsk. Astrofiz. Obs. 52, 49.

Nissen, P. E.: 1974, Astron. Astrophys. 36, 57.

Rogerson, J. B. and Lamers, H. J. G. L. M.: 1975, Nature 256, 190.

Rogerson, J. B. and Lamers, H. J. G. L. M.: 1976, 250ème colloque international du CNRS, Physique des mouvements dans les atmosphères stellaires, Nice.

Underhill, A. B.: 1975, Phil. Trans. Roy. Soc. London A279, 429.

\section{B. Helium-rich and Hydrogen-deficient Stars}

Clas-Offick, I.: 1976, Mitt. Astron. Ges. (in press).

Dufton, P. L.: 1973, Astron. Astrophys. 28, 267.

Higginbotham, N. A. and Lee, P.: 1974, Astron. Astrophys. 33, 277.

Hunger, K. and Kaufmann, J. P.: 1973, Mitt. Astron. Ges. 32, 241.

Kaufmann, J. P. and Hunger, K.: 1975, Astron. Astrophys. 38, 351.

Kaufmann, J. P. and Schoenberner, D.: 1976, Mitt. Astron. Ges. (in press).

Osmer, P. S. and Peterson, D. M.: 1974, Astrophys. J. 187, 117.

Schoenberner, D.: 1973, Astron. Astrophys. 28, 433.

Schoenberner, D. and Wolf, R. E. A.: 1974, Astron. Astrophys. 37, 87.

Wolf, R. E. A.: 1973, Astron. Astrophys. 26, 127.

\section{Ap, Am and A Stars}

Boesgard, A. M., Praderie, F., Leckrone, D. S., Faraggiana, R., and Hack, M.: 1974, Astrophys. J. Letters $194, \mathrm{~L} 143$.

Fracassini, M., Pasinetti, L. E., and Pelagatti, G.: 1975, Astrophys. Space Sci. 35, 313.

Khokhlova, V. L. and Rjabchikova, T. A.: 1975, Astrophys. Space Sci. 34, 403.

Little, S. J.: 1974, Astrophys. J. 193, 639.

Stickland, D. J.: 1975, IAU Coll. 32, Vienna.

Zverko, J.: 1974, Bull. Astron. Inst. Czech. 25, 34.

Zverko, J.: 1975, Bull. Astrophys. Inst. Czech. 26, 58.

\section{Solar-type Stars}

Blackwell, D. E., Ellis, R. S., Ibbetson, P. A., Petford, A. D., and Willis, R. B.: 1975, Monthly Notices Roy. Astron. Soc. 171, 425 .

Cayrel de Strobel, G. and Pasinetti, L. E.: 1975, Astron. Astrophys. 43, 127.

Da Silva, L.: 1975, Astron. Astrophys. 41, 287.

Griffin, R.: 1975, Monthly Notices Roy. Astron. Soc. 171, 181.

Gustafsson, B., Kjaergaard, P., and Andersen, S.: 1974, Astron. Astrophys. 34, 99.

Hearnshaw, J. B.: 1973, Astron. Astrophys. 29, 165.

Hearnshaw, J. B.: 1974, Astron. Astrophys. 34, 263.

Hearnshaw, J. B.: 1975, Astron. Astrophys. 38, 271.

Helfer, H. L. and Jennens, P. A.: 1975, Monthly Notices Roy. Astron. Soc. 172, 701.

Mäckle, R., Griffin, R., Griffin, R., and Holweger, H.: 1975, Astron. Astrophys. Suppl. 19, 303.

Mäckle, R., Holweger, H., Griffin, R., and Griffin, R.: 1975, Astron. Astrophys. 38, 239.

Perrin, M. N.: 1975, Astron. Astrophys. 44,9.

Spite, F. and Spite, M.: 1975, Astron. Astrophys. 40, 141.

Williams, P. M.: 1973, Monthly Notices Roy. Astron. Soc. 162, 235.

\section{E. Very Late-type Stars}

Bell, R. A. and Dickens, R. J.: 1974, Monthly Notices Roy. Astron. Soc. 166, 89.

Boyarchuk, M. E.: 1972a, Izv. Krymsk. Astrofiz. Obs. 46, 54.

Boyarchuk, M. E.: 1972b, Izv. Krymsk. Astrofiz. Obs. 46, 47.

Boy archuk, A. D.: 1974, IAU Symp. 66, 198.

Bues, I.: 1973, Mitt. Astron. Ges. 32, 234.

Day, R. W., Lambert, D. L., and Sneden, C.: 1973, Astrophys. J. 185, 212. 
Johnson, H. R. and Fay, T. D.: 1973, Astrophys. J. 181, 851.

Lambert, D. L., Dearborn, D. S., and Sneden, C.: 1974, Astrophys. J. 193, 621.

Lambert, D. L. and Tomkin, J.: 1974, Astrophys. J. Letters 194, L89.

Lee, P.: 1974, Astrophys. J. 192, 133.

Querci, F., Querci, M., and Tsuji, T.: 1974, Astron. Astrophys. 31, 265.

Querci, F. and Querci, M.: 1975a, Astron. Astrophys. 39, 113.

Querci, M. and Querci, F.: 1975b, Astron. Astrophys.

Shavrina, A. V. and Yakovina, L. A.: 1973, Astrometrya i Astrofizika. No. 19, 87.

Shavrina, A. V.: 1974, Astrometrya $i$ Astrofizika, No. 20, 10.

Tomkin, J. and Lambert, D. L.: 1974, Astrophys. J. 193, 631.

Thompson, R. I.: 1973, Astrophys. J. 184, 187.

Thompson, R. I. and Johnson, H. L.: 1974, Astrophys. J. 193, 147.

Uus, U.: 1974, IAU Symp. 66, 122.

Williams, P. M.: 1975, Monthly Notices Roy. Astron Soc. 170, 343.

R. CAYREL

President of the Commission
D. MIHALAS

Vice-President of the Commission 